Although too few data are available for conclusive remarks, mosaic patients with various proportions of normal and abnormal cell lines and different clinical signs including immunological peculiarities may be useful in solving the mechanism of hyperblastic lymphocyte reaction in Down's syndrome.

\section{MOTOMTCHI SASAKI} Yoshitaka Obara

Zoological Institute, Hokkaido University, Sapporo, Japan.

Received December 23, 1968.

' Bain, B., Vas, M. R., and Lowenstein, L., Blood, 23, 108 (1964),

'Hirschhorn, K., Bach, F., and Kolodny, R. L., Science, 142, 1185 (1963).

'Hayakawa, H., Matsui, I., Higurashi, M., and Kobayashi, N., Lancet, i, 95 (1968)

\section{APPLIED SCIENCE}

\section{Reciprocating Superconducting Generator/motor}

WE report here a new approach to the use of superconductors in electrical machines (British Patent Application No. 1686/69). There are several advantages in using a superconductor instead of a conventional conductor in rotating electrical machinery.

First, in the field winding, a superconductor without iron can produce a much greater magnetic flux density than a normal conductor plus iron. (Copper alone could theoretically produce as great a flux density but at a quite prohibitive energy dissipation.) This should allow a reduction in weight, size and cost. The higher flux density also means that the induced e.m.f. per unit length of armature conductor is greater, which implies that for the same power, the size of armature, the rotational speed or the current may be reduced, either together or separately. If the armature rotates entirely within the uniform field of a superconducting solenoid, armature iron is not necessary, which contributes to further weight reduction.

Finally, the use of a superconductor in the field winding eliminates the $I^{2} R$ losses inevitable in a normal conductor. In a homopolar d.c. machine the losses in the armature are also eliminated if it is superconducting.

There are two principal types of machine having armatures rotating entirely within a solenoidal field winding. One is the homopolar machine, using rotation about an axis parallel to the field and producing d.c., and the second is an a.c. machine, in which the rotation is about an axis at right angles to the field. The armature of either machine may use either a superconductor or a normal conductor.

The homopolar machine (due to Faraday) needs to have a segmented rotor in order to work at moderate

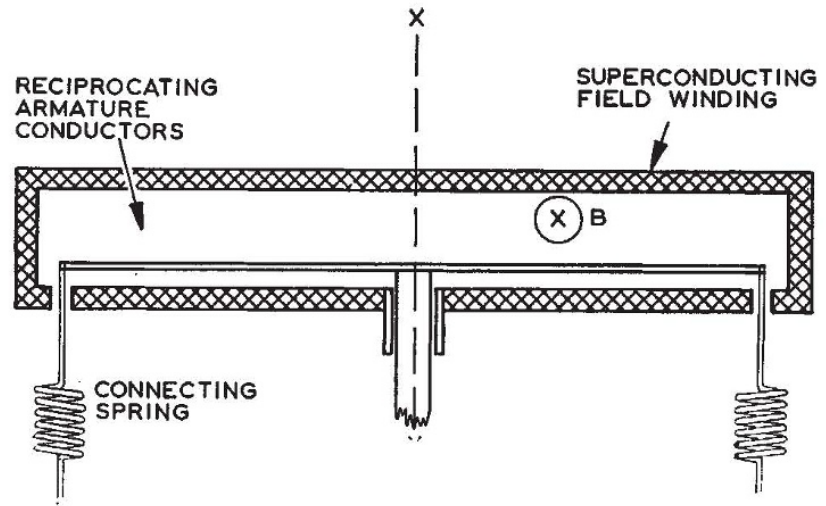

Fig. 1. Principle of reciprocating generator.

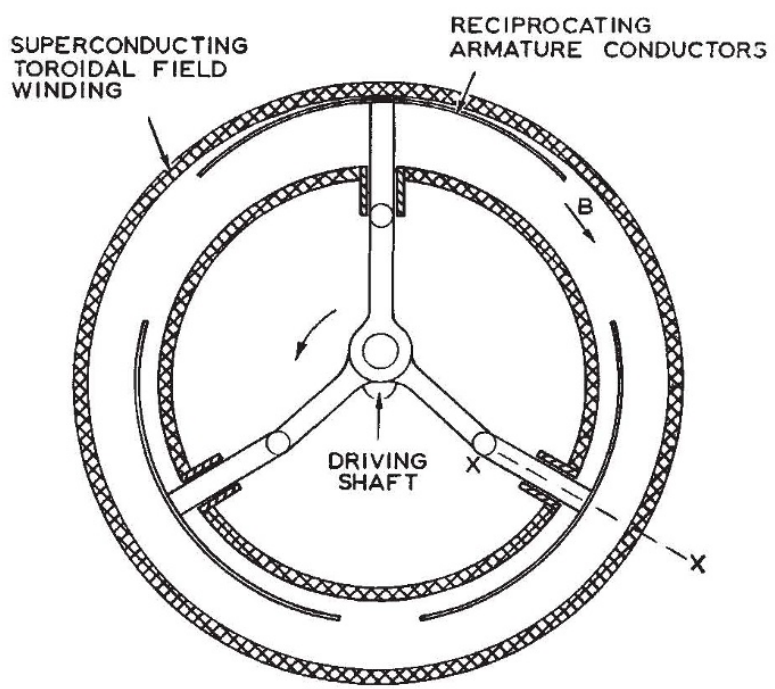

Fig. 2. End view of 3-phase generator.

voltage and current. The series connexion of the segments by contacts and brushes leads to difficulties in operation. Moreover, this machine has large stray fields from the open ends of the solenoid; it uses the field near the axis of the rotor only inefficiently because the linear speed is very small; and it can produce only d.c.

Although the a.c. machine has most of the advantages discussed above, it has the disadvantage of excessive a.c. loss resulting from the rotation of the armature in a high flux density (say, $50 \mathrm{kG}$ ). These losses (a few hundred $\mathrm{kW}$ in a $10 \mathrm{MW}$ machine) occur in both superconductors (as hysteresis losses) and normal conductors (eddy-current losses) and are additional to those resulting from the alternating current in the armature conductors; they can be greater than the Joule losses in a conventional machine handling the same power.

If, instead of rotating, the armature conductors reciprocate in the magnetic field, as shown in Fig. 1, an alternating e.m.f. is induced without excessive a.c. losses. Im. mersed in a unidirectional magnetic field, the a.c. losses now result from the current alone and they can be made acceptably small. Electrical connexion to the ends of the conductors need not be through sliding contacts; springs or flexible connexions can be used instead. Power can be transmitted to or taken from a shaft by means of a crank and connecting rod. Although such a machine can operate in a single-phase mode, with the armature oscillating across the field of a straight solenoid, the preferred operation is three-phase, using a toroidal field winding, as shown in Fig. 2. This arrangement has no stray magnetic field. Fig. 1 now becomes a representational section through $\mathrm{X}-\mathrm{X}$. Only three conductors are shown in the diagram, but each of these may be broken down to give several conductors per phase and these can then be connected in series outside the magnetic field to give, within limits, any required e.m.f. Although the field winding would certainly be superconducting, it has not yet been decided whether the armature should consist of super. conductors or normal conductors.

Because all the magnetic iron-which accounts for about half the weight of a conventional machine-is eliminated in the new design there would be a substantial weight saving. Preliminary calculations also indicate that, even with a very large machine ( $500 \mathrm{MW}$ ), some reduction in both size and power loss could be achieved.

\section{R. V. HARROWELL}

Central Electricity Research Laboratories,

CEGB,

Leatherhead, Surrey. 